\title{
Association of Natural Antioxidants and Immunity in Covid-19 Pandemic
}

\author{
Anjali Ambadas Vagga ${ }^{1}$, Lata Kanyal Butola², Shivram G. Khadhe³ ${ }^{3}$ Komal Ajay Meshram \\ 1,2 Department of Biochemistry, Jawaharlal Nehru Medical College, Datta Meghe Institute of Medical Sciences, Wardha \\ Maharashtra, India. ${ }^{3}$ Department of Cytogenetic, Smt. Kasturbai Walchand College, Sangli, Maharashtra, India. \\ ${ }^{4}$ Department of Physiology, Datta Meghe Medical College, Wanadogari, Nagpur, Maharashtra, India.
}

\section{ABSTRACT}

\section{BACKGROUND}

A pandemic is when an infectious disease occurs in different parts of the world simultaneously and quickly spreads from person to person. The pandemic of the $21^{\text {st }}$ century in the entire world is infection with the coronavirus. India now has the world's second-largest number of confirmed cases, next to US, following a recent increase in recorded infections. In case of serious corona infections, the out-ofcontrol immune system ultimately causes the patients' lungs to stop supplying oxygen to the body leading to respiratory failure. In some cases, the malfunctioning immune response in Covid-19 patients can drive the rapid decline in lung function. Symptoms of Covid-19, such as flu (influenza infection) similar to cold, can be avoided or mitigated and are mostly managed by combining a balanced diet with exercise and addition of antioxidants; the most common antioxidant is vitamin $\mathrm{C}$ in some form like raw or ripe fruits and vegetables is preferred and supplementations are also advised. Ascorbic acid is best known for its antioxidant properties and can scavenge damaging reactive oxygen species, thus protecting the body's cells and tissues from oxidative harm and dysfunction. However, vitamins also have many other significant body functions, many of which are known to promote good immune function. Vitamin C levels may be reduced during infection, and the demand for vitamin $\mathrm{C}$ enhances with the severity of the infection. Consequently, a possible antioxidant therapy may be suggested to get relief from the Covid-19 respiratory infection. An effective immune response relies on an appropriate diet and natural antioxidants, to hold infection at bay.

\section{KEY WORDS}

Natural Antioxidants, Vitamin C, Immunity, Covid-19, Pandemic
Corresponding Author: Anjali Ambadas Vagga, Jawaharlal Nehru Medical College, Datta Meghe Institute of Medical Sciences, Wardha Maharashtra, India.

E-mail: anjalivagga@gmail.com

DOI: $10.14260 / j e m d s / 2021 / 132$

How to Cite This Article:

Vagga AA, Butola L, Khadhe SG, et al. Association of natural antioxidants and immunity in covid-19 pandemic. J Evolution Med Dent Sci 2021;10(09):613-618, DOI: 10.14260/jemds/2021/132

Submission 24-09-2020,

Peer Review 02-01-2021,

Acceptance 09-01-2021,

Published 01-03-2021.

Copyright (C) 2021 Anjali Ambadas Vagga et al. This is an open access article distributed under Creative Commons Attribution License [Attribution 4.0 International (CC BY 4.0)] 


\section{BACKGROUND}

Coronavirus continues to spread worldwide, with more than 27 million cases confirmed in 188 countries. About 890,000 people have already lost their lives. Number of cases of the disease in many countries continue to surge. Those who have had obvious success in suppressing initial outbreaks have observed infections might occur again. The number of confirmed cases exceeded during spring, since extensive testing was not done in the first trimester of year in many countries. As of now, since late December 2019 it became easier to track the initial case and successive outbreak form a group of unusual cases of pneumonia in people in the Wuhan city of China. Symptom were first noticed in the early days of December 2019.1,2 Thus, Covid-19 originally originated in China, with the virus developing for four months and quickly spreading as a global threat to other countries worldwide.

On 12 January 2020, the World Health Organization (WHO) briefly named the new 2019 novel coronavirus (2019nCoV) virus, and on 12 February 2020 formally named this infectious coronavirus disease 2019 (Covid-19). The International Committee for Virus Taxonomy (ICTV) later officially designated the causative agent as SARS-CoV-2 on the basis of phylogeny, taxonomy and existing practice. ${ }^{3}$ India now has the world's second-largest number of confirmed cases, behind the US, following a recent increase in recorded infections. Newly reported cases reached 90,000 a day. India saw cases rising by nearly two million in August 2020, the largest single-month increase recorded during the pandemic anywhere in the world. The boom comes as the government continues to loosen restrictions to try to stimulate the economy but also represents increased testing-as regular testing has risen to over a million. The disease spread quickly around the world throughout the first months of 2020, and the WHO declared a global pandemic on 11 March after 1918 Spanish flu (H1N1), 1957 Asian flu (H2N2), 1968 Hong Kong flu (H3N2) and 2009 Pandemic flu (H1N1), resulting in an estimated 50 million, 1.5 million, 1 million and 300000 deaths, respectively. $.5,6,7$ Symptoms of Covid-19, such as flu (influenza infection) are similar to cold, can be avoided or mitigated and are mostly managed by combining a balanced diet with exercise and likely additional antioxidants; the most common is vitamin $\mathrm{C}$ in some form like raw or ripe fruits and vegetables are preferred and supplementations are also better. In general, many safe people contacted Covid-19 and got it back. It has been contacted by many more with high immunity and mild symptoms, but many people with poor immunity have contacted it and had more extreme symptoms, but never knew they had it until after recovering from Covid-19. This is similar to cases of influenza infection. Those at risk need to protect themselves from infections such as influenza or pneumonia, and take appropriate measures to remain safe and healthy. Once again, even if you are at risk, the main contribution to health is a healthy diet of fresh fruit, vegetables and exercise. ${ }^{8}$

\section{ANTIOXIDANTS}

Antioxidants are compounds that can interact safely to neutralize or end the chain reaction with free radicals before damage to essential molecules. They exert their mechanisms of defense in many ways - 1) Metabolic reactions that catalyze reactive oxygen species (ROS) reduction, e.g., catalase and superoxide dismutase. 2) Antioxidants that donate oxygen to free radicals, such as vitamins 3 ) Proteins that decrease prooxidant supply, such as haptoglobin, haemopexin, transferrin, and metallothionine. 4) Proteins, such as heat shock proteins that protect biomolecules via other processes. ${ }^{9,10}$

The endogenous mechanism of body protection in opposition to these free radicals plays a vital function that can be further enhanced by supplementing the dietary antioxidants. Antioxidants can usually be classified into two main groups, artificial and intrinsic. Main selected location of the damage to these free radicals and the body's protective response to antioxidants is at cellular stage. Depending on this, antioxidants can even be categorized as enzymatic antioxidants and non-enzymatic ones. Enzymatic antioxidants comprise mainly glutathione peroxidase, dismutase, and catalase with superoxide. The body also has many other enzymes that confer to the overall antioxidant potential which is expressed in the serum. ${ }^{11}$

Non-enzymatic antioxidants include vitamins such as retinol, tocopherol, ascorbic acid, and, to a lower degree, vitamin D, peptides, co-enzyme Q10 and certain elements (selenium and zinc) in many subdivisions. Polyphenolic compounds, reported to have an important antioxidant capacity, are the main ingredients from natural sources. ${ }^{12}$

Phenolics occurring in every part of plants are mainly natural antioxidants. Some toxicological research involving the utilization of artificial antioxidants has manifested their unintended or harmful effects in the recent past. Such findings have encouraged the researchers to concentrate their analysis on evaluating the micronutrients with sufficient antioxidant capacity. ${ }^{13}$ In addition, the use of these natural antioxidants, supply and the economy are also relevant considerations. Natural antioxidants may be classified into different sub classifications.

However, two main types are antioxidants from frequently consumed or daily organic foods (e.g., veggies, grains, beans and fruits) and from plant or natural sources with sufficient antioxidant capacity, but not from daily organic foods. (e.g., medicinal plants and wild plants). Of these, the readily available and more suitable for dietary interferences, regular organic food are very relevant. The need is to recognize and create awareness of the above channels that could be appraised from peak to bottom for antioxidant capacity. Individuals consuming these fruits and vegetables daily in meals are less affected by various chronic diseases, ${ }^{14}$ and the long-term health benefit of consuming these diets of natural sources has also been endorsed by studies. The most common dietary supplements are both synthetic and natural vitamin $\mathrm{C}$ and E sources.

The highly recommended dietary content of fruit and vegetables is well known for its nutritious and healthpromoting effects. Due to their excessive mineral content, especially electrolytes; vitamins, ascorbic acid (C) and alpha tocopherol (E) in particular; although different resent research shows their biochemical content with antioxidant potentials. ${ }^{15}$ Nearly all-natural antioxidants transform the lipid radicals into more firm products after breaking the chain. Antioxidants extracted from fruits and vegetables are predominantly phenolic structures that can contain vitamins, polyphenols and minerals. ${ }^{16}$ Antioxidant elements, such as 
iron, selenium, zinc, copper and manganese, serve as a cofactor for most of the antioxidant enzymes, the lack of which will definitely disrupt their enzymatic scavenging activity. ${ }^{17}$

\section{VITAMIN C}

Vitamin C, that is ascorbic acid, is highly active water-soluble antioxidant typically present in citrus fruits such as oranges, lemons ${ }^{18}$ and vegetable fruits like tomatoes. ${ }^{19}$ Vegetables and fruits high in ascorbic acid should be consumed in low doses rather than at high doses at the same time as ascorbic acid shows less assimilation when consumed in large amounts. ${ }^{20}$

The mango peel contains a variety of antioxidants, like carotenoids, vitamin C, phenolic compounds, and dietary fiber. These substances demonstrate efficacy against many chronic diseases, such as Alzheimer's disease, Parkinson's disease, cancer and cataract. ${ }^{21}$ Vitamin $C$ in the aqueous phase of cells is an important antioxidant; it simply sheds off electrons to give stability to reactive oxygen species such as ROS. ${ }^{22}$ Besides the biological functions of vitamin $\mathrm{C}$ as superoxide and hydroxyl radical remover, it also acts as cofactor. ${ }^{23}$ Vitamin $\mathrm{C}$ plays a key role in protecting against oxidative damage, especially in leukocytes, and can influence chronic degenerative diseases, autoimmune diseases and cancer treatment. 22,23

\section{Benefits of Vitamin C}

For several years, vitamin $\mathrm{C}$ has been extensively researched and known to be an effective cofactor that is required in the development of bone, blood vessels, muscles, animal tissues and is necessary for the repair mechanism. The vitamin, as an antioxidant, ought to assist shielding cells from chemical-free radical injury. These chemicals are known to lead to cancer, heart disease and other illnesses. Decreasing endothelial dysfunction, managing blood pressure, minimizing the risk of heart disease and averting stroke, ascorbic acid also has beneficial effects on certain types of chronic disorders. The interpretation of these possible benefits is often disputable and / or contradictory and it is exactly indefinite that the advantages are at least partly the outcome of a healthy diet. Overall, frequent intake of vitamin $\mathrm{C}$ additives minimizes the acute rhinitis period but does not minimize the risk of catching cold except for people under serious physical strain (e.g. marathoners, ski jumpers, or soldiers in subarctic conditions) where so ever cold frequency is halved. Ingesting vitamin $\mathrm{C}$ additives after the onset of cold symptoms have already started shows no significant benefits. ${ }^{24}$ The antiviral activity is likely to be the reason why vitamin $\mathrm{C}$ has aroused attention to be a potential Covid-19 therapy. The maximum per day intake of ascorbic acid allowance is 0.09 gm per day for adult males, $0.075 \mathrm{gm}$ for adult females, an additional 0.035 gm daily for smokers of both sexes. ${ }^{24}$ "The Linus Pauling Institute" suggests a per day ascorbic acid consumption of at least $0.4 \mathrm{gm}$ for adults > 50 years. The dosage advice from Dr. Pauling indicated that the optimal daily dose should be around $2 \mathrm{gm}$, he also illustrated that the initial $0.250 \mathrm{gm}$ is most crucial for reaching sufficient blood concentration. ${ }^{25}$

\section{Role of Vitamin C during Covid-19 Infection}

During his 31 years of practice, Dr. Hunning Hake records inoculating intravenous vitamin $C$ to 250,000 patients and defines ascorbic acid as "the supreme rescue molecule in nature." His recent procedure is to inoculate $7500 \mathrm{mg}$ of ascorbic acid in fifty $\mathrm{ml}$ fluid in about 35 minutes and victims accept this remedy quite well, with absolutely no visible kidney stone growth. Vitamin C administration in patients with Covid-19 infection has been implemented by at least some healthcare systems. A New York (NY) newspaper notes that vitamin $C$ is given to chronically ill victims in NY City's largest hospital system. Dr. Andrew G. Weber, a pulmonologist and critical care physician, observed that his coronavirus critical-care victims bear $1500 \mathrm{mg}$ of vitamin C immediately, given intravenously, and the dosage is repeated 3 to 4 times daily. Dr. Weber said, "Patients receiving vitamin $\mathrm{C}$ have shown better results than those who were not administered with vitamin C," Because Covid-19 patients' levels of vitamin C drop significantly as septicaemia occurs, Dr. Weber further states, "Trying to preserve vitamin C levels in the body makes good sense all over the world." Another statement from a representative from Northwell operating $23 \mathrm{NY}$ hospitals asserts vitamin $C$ should be "widely used" all (over) the system with varying procedures as per the physician's orders. ${ }^{26} \mathrm{~A}$ double-blind, dummy-controlled study is required to determine conclusively whether vitamin $\mathrm{C}$ remedy is effective in Covid-19 treatment or not. Indeed, such study has been planned and on February 14, $2020^{27}$ the recruitment of subjects started. For patients showing serious pneumonia due to Covid-19, the study plan demands for IV injection of either $12000 \mathrm{mg}$ of ascorbic acid or dummy medicine twice a day for 7 days.

A new research report conducted in 2019 prior to the discovery of the Wuhan coronavirus offers a systematic review of 18 controlled clinical trials with a total of more than two thousand patients assessing the effect of vitamin $\mathrm{C}$ on the length of stay in the hospital's critical care unit and the time of ventilation assisted. The findings in the analysis comprised the following: In 12 of the studies analysed, the duration of ICU stay in patients getting vitamin $\mathrm{C}$ was decreased by 7.8 percent. That was statistically highly important. The period of ICU stay was decreased by 8.6 percent in six studies after vitamin C given orally at doses of $1-3 \mathrm{~g} /$ day. During 3 different studies where patients required assisted ventilation for vitamin C during 24 hours, the mechanical ventilation period was shortened by $18.2 \%$. All these data were of great statistical significance. ${ }^{28}$

Vitamin C can be helpful in managing SARS-CoV-2 due to its antioxidant effect, antiviral properties, immune system boosting capability and anti-inflammatory properties. Vitamin $\mathrm{C}$ may also help to eliminate the alveolar fluid that accumulates during acute respiratory distress syndrome (ARDS) by preventing activation and aggregation of neutrophils and by reducing alveolar epithelial damages. Intravenous infusion of up to $100 \mathrm{~g}$ is safe, provided the above listed precautions are taken. ${ }^{29}$

\section{Role of Vitamin C in Immunity}

Vitamin $C$ regulates immune functions by modulating redox sensitive cell signaling pathways or by instantly safeguarding essential structural components of the cell. Neutrophils' 
manifestation to oxidation, for example impair cell movement, that is believed to be due to membrane lipid oxidation resulting in an impact on fluidity of cell membrane. ${ }^{30}$ In vitro studies have shown that in presence of vitamin C, lymphocytes enhance multiplication ${ }^{31,32}$ subsequently there is an increased antibody output which defends different cell death stimuli. ${ }^{33}$ In addition, ascorbic acid plays a crucial role in the developmental differentiation and maturation of immature $\mathrm{T}$ cells $^{31}$ with mature and immature NK (Natural Killer) cells related multiplication and maturation effects have been observed. ${ }^{34}$

\section{Vitamin $C$ in Other Infections}

Victims with acute respiratory infections like pneumonia and pulmonary tuberculosis show reduced plasma vitamin $\mathrm{C}$ concentrations compared to control volunteers. ${ }^{35}$ In patients having acute respiratory infections, vitamin $\mathrm{C}$ administration restores their normal vitamin $\mathrm{C}$ plasma levels and improves the rigidity of the breathing symptoms. ${ }^{36}$ Cases of acute infections of the lungs demonstrate rapid chest X-ray evacuation following intravenous vitamin C. 37,38 The ascorbic acid dependent evacuation of neutrophils from lung infection might possibly attribute to intense apoptosis and subsequent phagocytosis and macrophages removal of consumed neutrophils. ${ }^{39}$ Preclinical studies of animals with septicaemiainduced lung damage have shown ascorbic acid dose to be capable of raising alveolar fluid clearance, enhancing broncho alveolar epithelial barrier efficiency, and attenuating neutrophil segregation, ${ }^{40}$ all important factors for normal lung function. By improving different immune cell functions, vitamin $\mathrm{C}$ appears to help inhibit and heal respiratory and systemic infections. Inhibition of infection includes dietary consumption of ascorbic acid which provide at least sufficient, if not saturated, blood concentration (i.e., $0.1-0.2 \mathrm{gm} /$ day) that maximize the levels of cells and tissues. In comparison, treatment of known infections needs substantially larger doses of vitamins to countervail for increased metabolic demand. 41

\section{IMMUNITY IN HEALTHY SYSTEM}

Immunity mainly involves the resistance shown and protection offered by host organism against the infectious diseases. Immunity is categorised into two types-innate immunity and acquired immunity. The immune system represents the third and most potent defence mechanism of the body. The B-cells possess the capability to specifically recognise each antigen and produce immunoglobulins against it. When an infection takes place or when an antigen enters the body, it is trapped by the macrophages in lymphoid organs. The phagocytic cells which are guarding the body by constant patrolling engulf and digest foreign substances. However, the partially digested antigenic epitopes attach to lymphocytes.

\section{IMMUNITY IN COVID-19}

Our immune system is the best protection since there is no recorded medication or vaccine to treat Covid-19. The body tries its best to overcome various infectious agents like bacteria, virus, protozoa and fungi.

In the first line of defence, skin provides mechanical barrier to prevent the entry of bacteria and viruses. Despite the physical barrier, the microorganisms do enter the body. The body defends itself and eliminates the invading organisms by non-specific mechanism like sneezing and secretions of mucus. The inflammatory response and fever response of the body and the immune system is inefficient then ultimately, we get ill. The same has happened in the case corona infection.

$\mathrm{T}$ cells help the $\mathrm{B}$ cells transform to plasma cells that produce antibodies specific for a viral antigen in return. Natural antibody neutralization succeeds in completely preventing the virus from entering host cells to reduce the infection playing a very severe defensive task at the later stage of infection and prevents recurrent infection. On the other hand, a cell-mediated immune response can be observed in infected cells that are mediated by T-lymphocytes. Helper T cells control the overall adaptive immune response, while cytotoxic $\mathrm{T}$ cells play a key role in clearing and removing viral infected cells. ${ }^{42}$ Treatment with vitamin $C$ can protect innate alveolar epithelial type II immunity (ATII) by inhibiting lactate secretion created by the activated immune cells.

Vitamin $\mathrm{C}$ is best known for its antioxidant properties and can scavenge harmful species of reactive oxygen, thus shielding the cells and tissues of the body from oxidative damage and dysfunction. The vitamin, however, also has many other significant body functions, many of which are known to promote good immune function. This may involve intravenous administration of doses in grams in extreme cases in order to reach sufficiently high levels in the body to compensate for the increased vitamin turnover.

Hemila and colleagues described that multiple high-dose intravenous VC infusions (e.g. $200 \mathrm{mg}$ / kg body weight / day, divided into 4 doses) decreased the duration of the intensive care unit (ICU) by $7.8 \%,{ }^{28}$ followed by a substantial reduction in the mortality rate. ${ }^{43}$ This experience has been repeated among serious influenza patients. 44 In reality, dietary antioxidants (VC and sulforaphane) have been shown to minimize oxidative stress caused by acute inflammatory lung injury in patients receiving assisted ventilation 45 In addition, oral VC (e.g. $6 \mathrm{~g}$ daily) might minimize the risk of viral infection ${ }^{46}$ or refine symptoms. ${ }^{47}$

High-dose intravenous VC is successfully used in 50 mild to extreme Covid-19 patients in China. The dosage used in treatment ranged from $10 \mathrm{~g}$ to $20 \mathrm{~g}$ per day, given over an 8$10 \mathrm{~h}$ period. In critical conditions, additional VC bolus can be needed between patients. The index of oxygenation increased in real-time and all patients were finally healed and relieved. ${ }^{48}$ In fact, for many decades high-dose VC has been clinically used and a recent National Institutes of Health (NIH) expert panel document clearly expresses that this protocol ( $1.5 \mathrm{~g} / \mathrm{kg}$ body weight) is safe and without significant terrible side effects. ${ }^{49}$

\section{CONCLUSIONS}

The unmanageable immune system ultimately causes the patients' lungs to stop supplying oxygen to the body in the event of serious corona infection, leading to respiratory failure. In some cases, malfunctioning immune response in Covid-19 patients can drive the rapid decline in lung function. 
Covid-19 symptoms, such as flu (influenza infection) and cold like symptoms can be avoided or mitigated and are mostly treated by the combination of a balanced diet and maybe additional antioxidants like vitamin $\mathrm{C}$, in some form like raw or ripe fruits and vegetables and adequate supplementation may also be given. A possible antioxidant therapy may therefore be suggested to reduce Covid-19 respiratory infection. In order to hold infection at bay, an effective immune response relies on an appropriate diet and natural antioxidants.

Financial or other competing interests: None.

Disclosure forms provided by the authors are available with the full text of this article at jemds.com.

\section{REFERENCES}

[1] Huang C, Wang Y, Li X, et al. Clinical features of patients infected with 2019 novel coronavirus in Wuhan, China. Lancet 2020;395(10223):497-506.

[2] Zhu N, Zhang D, Wang W, et al. A novel coronavirus from patients with pneumonia in China, 2019. N Engls J Med 2020;382(8):727-33.

[3] CSG of the International CS. The species severe acute respiratory syndrome-related coronavirus: classifying 2019-nCoV and naming it SARS-CoV-2. Nat Microbiol 2020;5(4):536-44.

[4] Johnson NPAS, Mueller J. Updating the accounts: global mortality of the 1918-1920 Spanish influenza pandemic. Bull Hist Med 2002;76(1):105-15.

[5] Kain T, Fowler R. Preparing intensive care for the next pandemic influenza. Critical Care 2019;23(1):337.

[6] Simonsen L, Clarke MJ, Schonberger LB, et al. Pandemic versus epidemic influenza mortality: a pattern of changing age distribution. J Infect Dis 1998;178(1):53-60.

[7] Viboud C, Simonsen L, Fuentes R, et al. Global mortality impact of the 1957-1959 influenza pandemic. J Infect Dis 2016;213(5):738-45.

[8] Slavin JL, Lloyd B. Health benefits of fruits and vegetables. Adv Nutr 2012;3(4):506-16.

[9] Vertuani S, Angusti A, Manfredini S. The antioxidants and pro-antioxidants network: an overview. Curr Pharm Des 2004;10(14):1677-94.

[10] Valko CM, Leibfritz D, Moncol J, et al. Free radicals and oxidative stress: oxidants and antioxidants. Exp Physiol 2000;82:291-5.

[11] Anwar H, Rahman ZU, Javed I, et al. Effect of protein, probiotic and symbiotic supplementation on serum biological health markers of molted layers. Poult Sci 2012;91(10):2606-13.

[12] Carocho M, Ferreira IC. A review on antioxidants, prooxidants and related controversy: natural and synthetic compounds, screening and analysis methodologies and future perspectives. Food Chem Toxicol 2013;51:15-25.

[13] Ramalakshmi K, Kubra IR, Rao LJ. Antioxidant potential of low-grade coffee beans. Food Research International 2008;41(1):96-103.

[14] Dembinska-Kiec A, Mykkanen O, Kiec-Wilk B, et al. Antioxidant phytochemicals against type 2 diabetes. $\mathrm{Br} \mathrm{J}$ Nutr 2008;99(E-S1):ES109-17.
[15] Slavin JL, Lloyd B. Health benefits of fruits and vegetables. Adv Nutr 2012;3(4):506-16.

[16] Hurrell RF. Influence of vegetable protein sources on trace element and mineral bioavailability. J Nutr 2003;133(9):2973S-7.

[17] Sonia NS, Mini C, Geethalekshmi PR. Vegetable peels as natural antioxidants for processed foods- a review. Agricultural Reviews 2016;37(1):35-41.

[18] McGhie TK, Walton MC. The bioavailability and absorption of anthocyanins: towards a better understanding. Mol Nutr Food Res 2007;51(6):702-13.

[19] Urquiaga IN, Leighton F. Plant polyphenol antioxidants and oxidative stress. Biol Res 2000;33(2):55-64.

[20] Daniel JW. Metabolic aspects of antioxidants and preservatives. Xenobiotica 1986;16(10-11):1073-8.

[21] Ayala-Zavala JF, Rosas-Domínguez C, Vega-Vega V, et al. Antioxidant enrichment and antimicrobial protection of fresh-cut fruits using their own byproducts: looking for integral exploitation. J Food Sci 2010;75(8):R175-81.

[22] Arredondo MLO, Betancourt MCP, Yoshida MLB, et al. Relationship between vitamin intake and total antioxidant capacity in elderly adults. Uni Sci 2016;21(2):167-77.

[23] Skowyra M. Antioxidant properties of extracts from selected plant materials (Caesalpiniaspinosa, Perillafrutescens, Artemisia annua and Viola wittrockiana) in vitro and in model food systems. Universitat Politècnica de Catalunya 2014.

[24] Linus Pauling Institute, Micronutrient Center, Vitamin C. https://lpi.ore gonstate.edu/mic/vitamins/vitamin-C. Accessed August 16, 2020.

[25] The Linus Pauling Institute, Dr. Pauling's recommendation.

https://lpi.oregonstate.edu/mic/vitamins/vitaminC/Pauling-recommendation. Accessed on Aug 16, 2020.

[26] Mongelli L, Golding B. New York hospitals treating coronavirus patients with vitamin C. New York Post Metro 2020.

[27] Peng Z. Vitamin C infusion for the treatment of severe 2019-nCoV infected pneumonia. Clinical Trials 2020. gov. https://clinicaltrials.gov/ct2/show/NCT04264533.

[28] Hemilä H, Chalker E. Vitamin C can shorten the length of stay in the ICU: a meta-analysis. Nutrients 2019;11(4):708.

[29] Hernández A, Papadakos PJ, Torres A, et al. Two known therapies could be useful as adjuvant therapy in critical patients infected by COVID-19. Rev Esp Anestesiol Reanim 2020; 67(5):245-52.

[30] Anderson R. Ascorbate-mediated stimulation of neutrophil motility and lymphocyte transformation by inhibition of the peroxidase/H2O2/halide system in vitro and in vivo. Am J Clin Nutr 1981;34(9):1906-11.

[31] Huijskens MJ, Walczak M, Koller $N$, et al. Technical advance: ascorbic acid induces development of doublepositive $\mathrm{T}$ cells from human hematopoietic stem cells in the absence of stromal cells. J Leukoc Biol 2014;96(6):1165-75.

[32] Molina N, Morandi AC, Bolin AP, et al. Comparative effect of fucoxanthin and vitamin $\mathrm{C}$ on oxidative and functional parameters of human lymphocytes. Int Immunopharmacol 2014;22(1):41-50. 
[33] Campbell JD, Cole M, Bunditrutavorn B, et al. Ascorbic acid is a potent inhibitor of various forms of $\mathrm{T}$ cell apoptosis. Cell Immunol 1999;194(1):1-5.

[34] Huijskens MJA, Walczak M, Sarkar S, et al. Ascorbic acid promotes proliferation of natural killer cell populations in culture systems applicable for natural killer cell therapy. Cytotherapy 2015;17(5):613-20.

[35] Bakaev VV, Duntau AP. Ascorbic acid in blood serum of patients with pulmonary tuberculosis and pneumonia. Int J Tuberc Lung Dis 2004;8(2):263-6.

[36] Hunt C, Chakravorty NK, Annan G, et al. The clinical effects of vitamin C supplementation in elderly hospitalised patients with acute respiratory infections. Int J Vitam Nutr Res 1994;64(3):212-9.

[37] Bharara A, Grossman C, Grinnan D, et al. Intravenous vitamin $\mathrm{C}$ administered as adjunctive therapy for recurrent acute respiratory distress syndrome. Case Rep Crit Care 2016;2016:8560871.

[38] Fowler AA, Kim C, Lepler L, et al. Intravenous vitamin C as adjunctive therapy for enterovirus/rhinovirus induced acute respiratory distress syndrome. World J Crit Care Med 2017;6(1):85-90.

[39] Vissers MCM, Wilkie RP. Ascorbate deficiency results in impaired neutrophil apoptosis and clearance and is associated with up-regulation of hypoxia-inducible factor 1alpha. J Leukoc Biol 2007;81(5):1236-44.

[40] Fisher BJ, Kraskauskas D, Martin EJ, et al. Mechanisms of attenuation of abdominal sepsis induced acute lung injury by ascorbic acid. Am J Physiol Lung Cell Mol Physiol 2012;303(1):L20-32.

[41] Carr AC, Maggini S. Vitamin C and immune function. Nutrients 2017;9(11):1211.
[42] Kumar S, Nyodu R, Maurya VK, et al. Host immune response and immunobiology of human SARS-CoV-2 infection. In: Coronavirus Disease 2019 (COVID-19). Singapore: Springer 2020: p. 43-53.

[43] Marik PE, Khangoora V, Rivera R, et al. Hydrocortisone, vitamin $\mathrm{C}$ and thiamine for the treatment of severe sepsis and septic shock: a retrospective before-after study. Chest 2017;151(6):1229-38.

[44] Gonzalez MJ, Berdiel MJ, Duconge J, et al. High dose vitamin $C$ and influenza: a case report. J Orthomol Med 2018;33(3).

[45] Patel V, Dial K, Wu J, et al. Dietary antioxidants significantly attenuate hyperoxia-induced acute inflammatory lung injury by enhancing macrophage function via reducing the accumulation of airway HMGB1. Int J Mol Sci 2020;21(3):977.

[46] Kim TK, Lim HR, Byun JS. Vitamin C supplementation reduces the odds of developing a common cold in Republic of Korea Army recruits: randomised controlled trial. BMJ Mil Health 2020 .

[47] Gorton HC, Jarvis K. The effectiveness of vitamin C in preventing and relieving the symptoms of virus-induced respiratory infections. J Manipulative Physiol Ther 1999;22(8):530-3.

[48] Cheng RZ. Can early and high intravenous dose of vitamin C prevent and treat coronavirus disease 2019 (COVID19)? Med Drug Discov 2020;5:100028.

[49] PDQ Integrative, Alternative and Complementary Therapies Editorial Board. High-dose vitamin c (pdq®): health professional version. In: PDQ Cancer Information Summaries. Bethesda (MD): National Cancer Institute (US) 2002. 